\title{
OCCURRENCE OF METACERCARIAE OF DIPLOSTOMUM AND TYLODELPHYS SPECIES (DIPLOSTOMIDAE) IN CLARIAS SPECIES (CLARIIDAE) FROM LAKE VICTORIA
}

\author{
MJ Musiba and G Nkwengulila \\ Department of Zoology and Marine Biology \\ P. O. Box 35064, Dar Es Salaam, Tanzania. \\ E-mail: gamba@udsm.ac.tz
}

\begin{abstract}
The localisation and abundance of the metacercariae of Diplostomum and Tylodelphys species among the clariids of Lake Victoria were investigated between April and December 2001. Four species viz. Clarias gariepinus (271), C. alluaudi (65), C. werneri (50) and C. liocephalus (47) were examined, but only $\mathrm{C}$. gariepinus was infected in the brain case and along the nasal cavity. Prevalence of metacercariae was high (>70\%). Prevalence and mean intensity were similar between male and female fish. Mean intensity increased with fish size and declined in larger fish for D. mashonense; but declined with the increase in fish size for Tylodelphys species. The metacercariae were over-dispersed and the variance to mean ratio curves behaved similarly to mean intensity. Possible factors leading to the observed infection patterns are discussed.
\end{abstract}

\section{INTRODUCTION}

Parallel with the development of fish culture, the importance of fish diseases and parasites in the fishery industry has been underlined. Parasites can affect fish health in intensive culture systems causing considerable losses to the fishery industry (Parperna 1980, Field and Irwin 1994).

In the northern hemisphere, Diplostomum species have attracted attention because of their pathogenicity on fingerlings on fish farms (Field and Irwin 1994, Chappell 1995). Hence their biology has been studied intensively (Stables and Chappell 1986, Field and Irwin 1994, Chappell 1995). The metacercariae of Diplostomum species have been recorded in over 150 fresh and brackish water fish (Sweeting 1974, Niewiadomska 1996). The genus Tylodelphys is closely related to Diplostomum. The adults of both species infect closely related birds as final hosts (Niewiadomska 1996). Similarly, the metacercarial stages often co-exist in the same fish (Burrough 1978, Mwita 2002, Musiba 2004). The abundance of metacercariae of both genera varies among fish host species and with the size of the host (Betterton 1974, Nkwengulila 1995).
Studies on Diplostomum species in Africa are few and only one is known on Tylodelphys species (Mwita 2002). Similarly, in Tanzania few studies are known on Diplostomum species, and all refer to one fish species, $C$. gariepinus (Nkwengulila 1995, Mwita 2002, Musiba 2004). Nkwengulila (1995) also reported Diplostomum species from Oreochromis species (Cichlidae). The aim of the present study was to establish if other Clarias species that co-exist with $C$. gariepinus are also infected with Diplostomum species. Thus, the occurrence, localisation, and abundance of Diplostomum species among four Clarias species (C. gariepinus, $C$. alluaudi, $C$. werneri, and $C$. liocephalus) in the Mwanza gulf were investigated.

\section{MATERIALS AND METHODS}

Collection and handling of fish and metacercariae

Fish were caught using gill nets, traps and baited long lines and handlines. Dead fish were transported, to the laboratory, in an icecooled box and live ones in buckets containing lake water, at the Tanzania Fisheries Research Institute (TAFIRI), 
Mwanza Centre. Fish were examined soon on arrival, after the weight, standard length, sex and maturity stage of each fish have been recorded. Fish were then decapitated and the cranium opened using a strong and sharp scalpel. Metacercariae were flushed from their localisation by squirting tape water using a pipette. Metacercariae were pipetted into a watch glass, washed, sorted and counted.

\section{Data analysis}

Data for prevalence and mean intensity with respect to sex and size of fish were analyzed by $\chi^{2}$ and Kruskal-Wallis tests, respectively (Zar 1996).

\section{RESULTS}

Occurrence, prevalence and mean intensity of metacercariae

Of the four Clarias species examined, only C. gariepinus was infected. Three metacercariae, Diplostomum mashonense and two species of Tylodelphys (designated 1 and 2) co-existed in front of the brain along the nasal cavity and the fat tissue covering the olfactory nerve. About $97 \%$ of C. gariepinus examined were infected by at least one of the three types of metacercariae. The prevalence of D. mashonense, Tylodelphys 1 and 2 was generally high, $97 \%, 74 \%$ and $94 \%$, respectively. The intensity of infection among individual fish was extremely variable (Table 1). The mean intensity of both D. mashonense and Tylodelphys 2 were higher than that of Tylodelphys 1.

Table 1; Occurrence of the metacercariae of Diplostomum mashonense and Tylodelphys species in 271 Clarias gariepinus

\begin{tabular}{lccccccc}
\hline Parasite & $\begin{array}{c}\text { No. } \\
\text { infected }\end{array}$ & $\begin{array}{c}\text { Prevalence } \\
(\mathbf{\%})\end{array}$ & $\begin{array}{c}\text { Mean } \\
\text { intensity }\end{array}$ & std & Range & Abundance & $\begin{array}{c}\text { Proportion } \\
\mathbf{( \% )}\end{array}$ \\
\hline $\begin{array}{l}\text { Diplostomum } \\
\text { mashonense }\end{array}$ & 262 & 96.7 & 619.5 & $\begin{array}{c}780 . \\
5\end{array}$ & $1-5024$ & 598.9 & 58.35 \\
$\quad \begin{array}{l}\text { Tylodelphys } \\
\text { sp. 1 }\end{array}$ & 202 & 74.5 & 76.7 & $\begin{array}{c}260 . \\
5\end{array}$ & $1-1800$ & 57.2 & 5.58 \\
$\begin{array}{l}\text { Tylodelphys } \\
\text { sp. 2 }\end{array}$ & 255 & 94.1 & 393.5 & 593 & $1-3872$ & 370.2 & 36.07 \\
Overall & 264 & 97.4 & 1053.5 & $\begin{array}{c}109 \\
9\end{array}$ & $1-5024$ & 1026.3 & 100 \\
\hline
\end{tabular}

In general, Tylodelphys 1 occurred in lower proportions than Tylodelphys 2 and $D$. mashonense (Table 1). The interactions between the pairs of these parasites are shown on Fig. 1. Heavy infections of any two species seldom occurred in the same fish, but light infections of one species could co-exist with light or heavy infections of the other. Tylodelphys 1 always occurred in lighter infections whereby more than 250 metacercariae could hardly be found together with $\geq 1000$ Tylodelphys 2 and / or $D$. mashonense. However, in some instances, heavy infections by $D$. mashonense and Tylodelphys $2(\geq 2500$ metacercariae) were found in the same fish. 

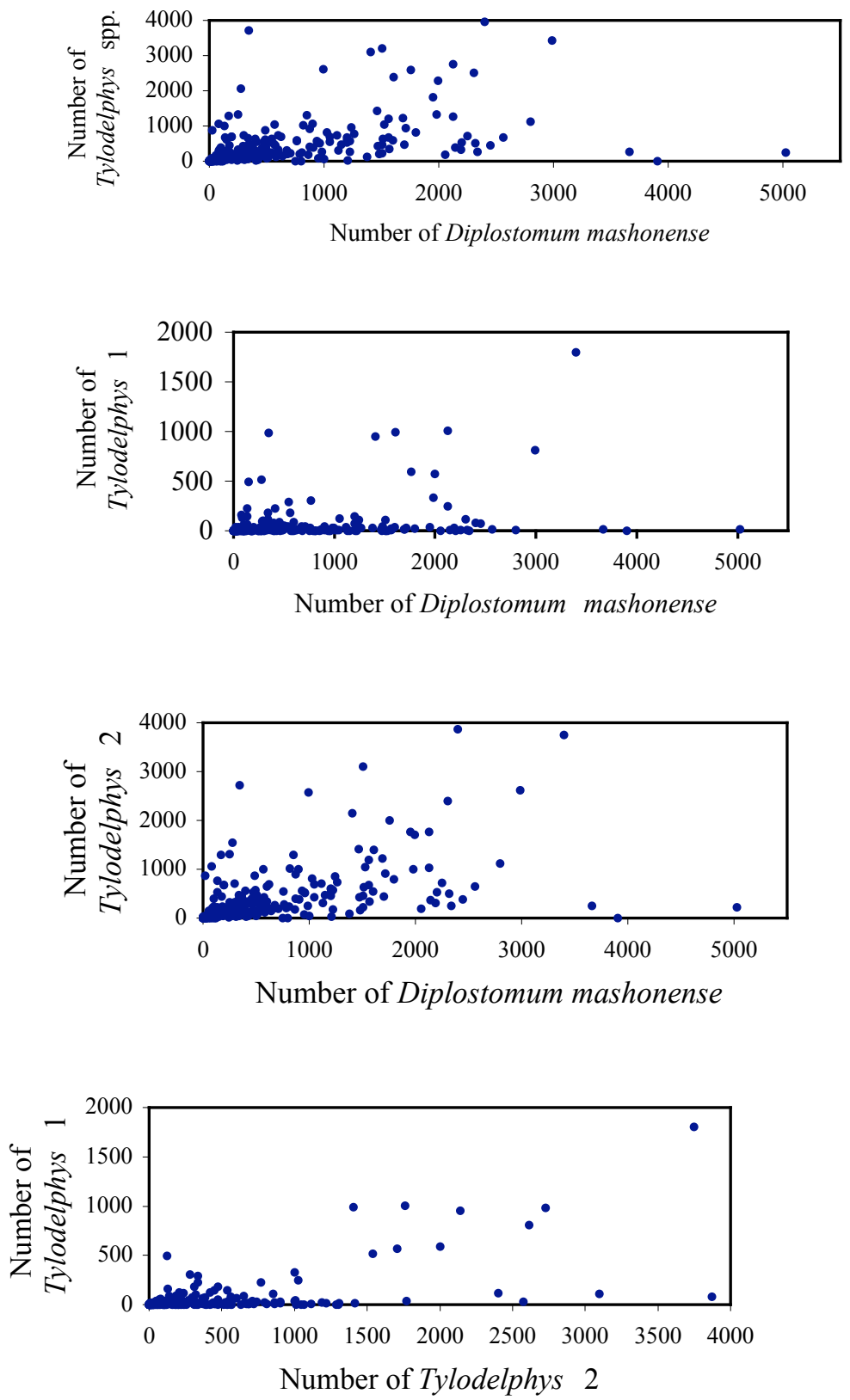

Figure 1: The relationship between the number of Diplostomum mashonense and Tylodelphys species 1 and 2 (pair wise comparisons) in the cranium of Clarias gariepinus (each dot represents one fish). 
Abundance of metacercariae in relation to sex and size of $C$. gariepinus

The prevalence and mean intensity of all the three metacercariae were not significantly different between female and male $C$. gariepinus (Table 2).

Table 2: $\quad$ Statistical test results for differences in the prevalence and intensity of Diplostomum and Tylodelphys metacercariae between male and female Clarias gariepinus

\begin{tabular}{lllll}
\hline Parameter & Test & D. mashonense & Tylodelphys sp 1 & Tylodelphys sp 2 \\
\hline Prevalence & $\chi^{2}$ & 1.77 & 3.09 & 1.85 \\
& $\mathrm{p}$ & 0.1833 & 0.0786 & 0.1732 \\
& Significance & NS & NS & NS \\
Mean & KW (H) & 10.63 & 11.29 & 10.07 \\
Intensity & p & 0.2235 & 0.1854 & 0.1732 \\
& Significance & NS & NS & NS \\
\hline
\end{tabular}

The prevalence of D. mashonense and Tylodelphys 2 was similar in all size classes with a slight decline in the $45-50 \mathrm{~cm}$ class: D. mashonense $\left(\chi^{2}=1.959, \mathrm{p}=0.9921\right)$ and Tylodelphys $2\left(\chi^{2}=1.860, \mathrm{p}=0.9935\right)$ (Fig. 2 ). The prevalence of Tylodelphys 1 was slightly low but reached $100 \%$ in the smallest and largest size classes, the variations were not statistically significant $\left(\chi^{2}=15.788, p=0.0714\right)$ (Fig. 2). The mean intensity of $D$. mashonense rose steadily with fish size but declined from fish of 20 $25 \mathrm{~cm}$ size class; these variations too were not statistically different $(\mathrm{H}=7.038, \mathrm{p}=$ 0.6332). The mean intensity of Tylodelphys
1 and Tylodelphys 2 decreased with fish size (Fig. 2), but again the variations were statistically not significant: Tylodelphys $1 \mathrm{(H}$ $=10.948, \mathrm{p}=0.2793)$ and Tylodelphys $2(\mathrm{H}$ $=6.634, \mathrm{p}=0.6751)$.

Frequency distribution of the intensity of metacercariae

Variance to mean ratio showed that both $D$. mashonense and Tylodelphys species were over dispersed (Fig. 3). The variance to mean ratio curves for all three species were similar to the mean intensity curves (Fig. 3 c.f. Fig. 2). 


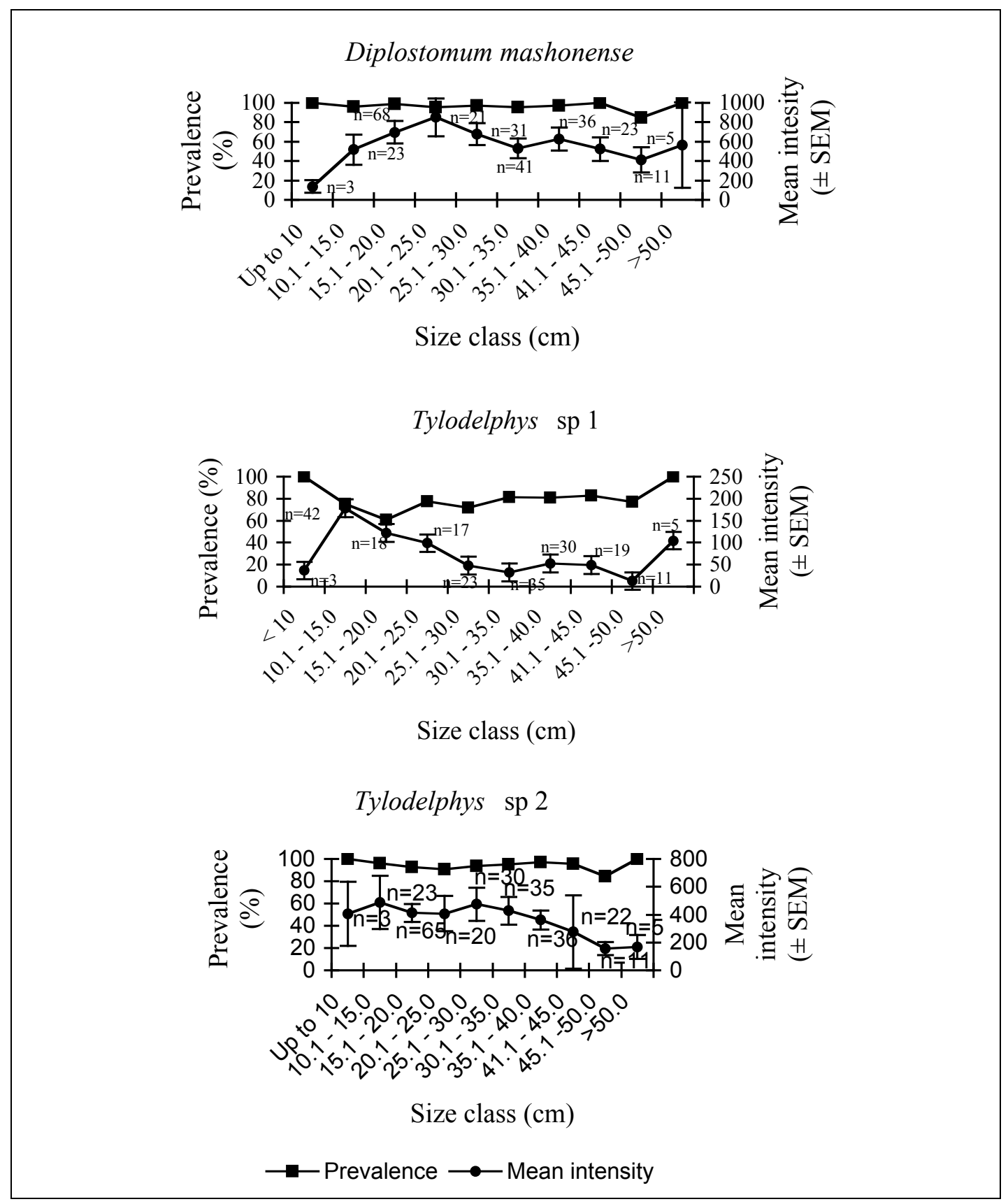

Figure 2: Prevalence and mean intensity of the three metacercariae in relation to the length of Clarias gariepinus. 


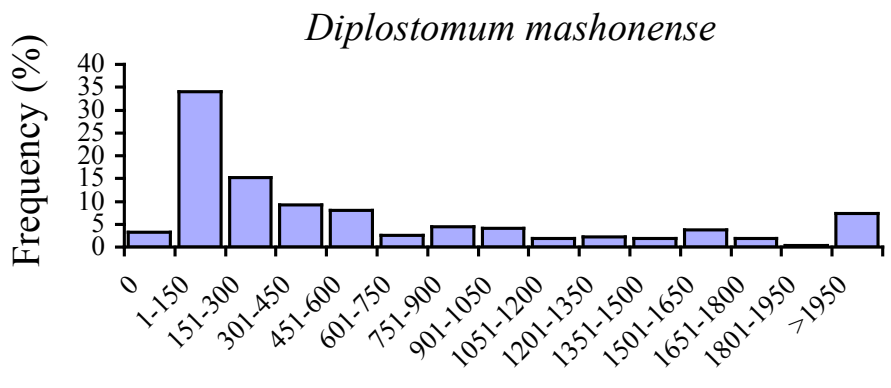

Number of metacercariae

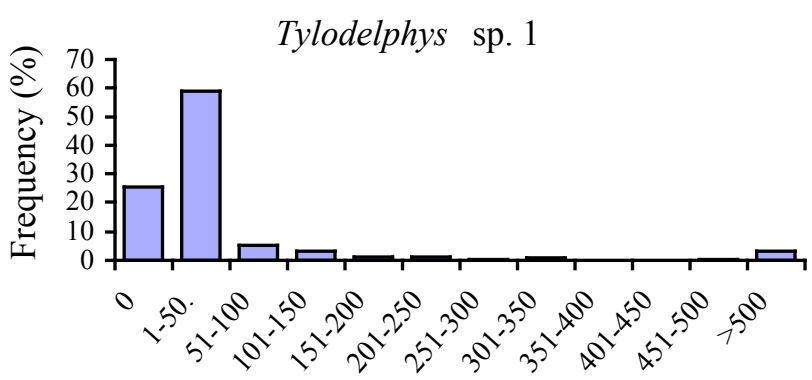

Number of metacercariae

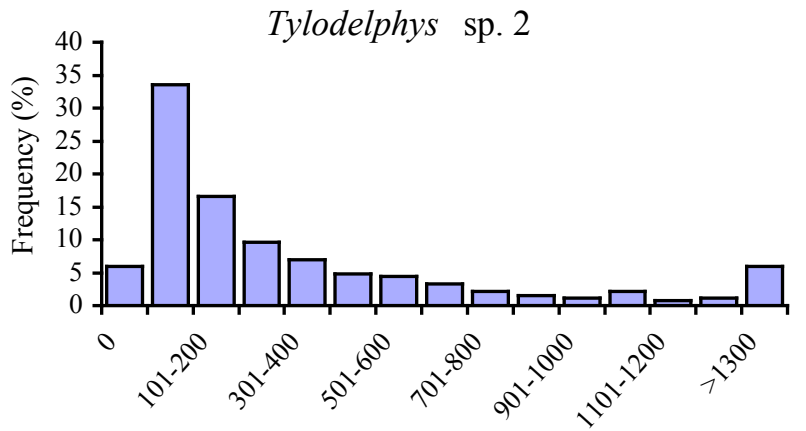

Number of metacercariae

Figure 3: $\quad$ Frequency distribution of Diplostomum mashonense and Tylodelphys species in Clarias gariepinus. 


\section{DISCUSSION \\ Occurrence, prevalence and mean intensity of metacercariae}

Metacercariae of $D$. mashonense were first reported by Beverley-Burton (1963) from the cranial cavity of $C$. gariepinus and $C$. ngamensis in Zimbabwe and Zambia, respectively. Prudhoe and Hussey (1977), and Mashego and Saayman (1989) recovered metacercariae of $D$. mashonense from the cranial cavity of $C$. gariepinus in South Africa. In Tanzania, metacercariae of $D$. mashonense have been reported in $C$. gariepinus from the Ruvu Basin (Nkwengulila 1995) and Lake Victoria (Mwita 2002). The earliest record of Tylodelphys species in Africa is that of adult worms from a kite, Buteo refofuscus augur Rüpp (Dubois and Fain 1956) from the Democratic Republic of Congo. The latest is by Mwita (2002) who recorded metacercaria of Tylodelphys species in C. gariepinus from the Mwanza Gulf, Lake Victoria. Nkwengulila (unpub. data) also reported Tylodelphys species in eyes of Oreochromis species and the cranial cavity of $C$. gariepinus from the Ruvu basin. The findings of the present study confirm the localisation and specificity of metacercariae of D. mashonense to $C$. gariepinus.

Doplostomum mashonense and Tylodelphys species were recorded from $C$. gariepinus but none in the other three clariids. Differential susceptibility of closely related fish species to metacercariae of Diplostomum species is well documented (Betterton 1974, Sweeting 1974, Burrough 1978, Nkwengulila 1995). Since the four Clarias species are closely related and inhabit similar habitats, the absence of infection in the other three Clarias species may most likely be due to failure of migrating cercariae to penetrate the tissues of the other three clariids. Other physiological factors, such as absence of stimuli or presence of conflicting stimuli, which disorient migrating cercariae, and a strong immune response may retard or hinder establishment within the host (Betterton 1974, Sweeting 1974).

The high prevalence (> 70\%) of $D$. mashonense and Tylodelphys species observed is supported by reports of earlier workers (Nkwengulila 1995, Mwita 2002) in Tanzania, and elsewhere in Africa (Beverley-Burton 1963, Mashego and Saayman 1989). The high infection statistics suggests that, $C$. gariepinus is highly susceptible to D. mashonense and Tylodelphys species.

There were always light infections of Tylodelphys 1 with heavy infections of $D$. mashonense and / or Tylodelphys 2 and that these two parasites (D. mashonense and Tylodelphys 2), were in some instances, found heavily infecting the same fish. There could be two explanations for this; either that heavy infection of D. mashonense and / or Tylodelphys 2 on the one hand, and those of Tylodelphys 1, is mutually exclusive. Alternatively, factors governing the infection processes prevent acquisition of large numbers of the two groups (i.e. $D$. mashonense and Tylodelphys 2, group 1 and Tylodelphys 1, group 2) of metacercariae (Kennedy and Burrough 1977, Burrough 1978). Heavy infections of D. mashonense and Tylodelphys species in the same fish suggest that these parasites share the same snail population as their first intermediate host (Machado et al. 1996). The snail intermediate hosts for Diplostomum and Tylodelphys species in Africa are not known. Elsewhere, lymnaeid species act as first intermediate hosts (Niewiadomska 1996).

\section{Abundance of metacercariae in relation to sex and size of Clarias gariepinus}

There were minor variations in prevalence and mean intensity between male and female fish. Other workers have reported similar 
observations (Shotter 1980, Nkwengulila 1995, Mwita 2002, Musiba, 2004), and this has been attributed to similar ecological relationships between female and male fish.

Mean intensity of $D$. mashonense increased with size but declined in larger fish, whereas that of Tylodelphys 1 and 2 decreased with size. Furthermore, few infected fish were $\leq$ $10 \mathrm{~cm}$ long, reflecting perhaps death of these smaller fish due either to massive cercarial penetration or by predation (Brassard et al. 1982). Fish size and age are known to be positively associated with abundance of metacercariae of diplostomids (Sweeting 1974, Nkwengulila 1995, Mwita 2002, Musiba, 2004). This has been attributed to; the long life span of metacercariae, continuous exposure of the fish to reinfection throughout their life and their inability to develop age related immunity.

The decline of intensity of infection in older fish might be due to low establishment rate caused by either development of immunity or behavioural changes in old fish, which result in low recruitment rate. Although evidence is lacking, it is possible that the parasites were dying within the fish. This would infer that the parasites' life span is short and that mortality rate exceeded recruitment rate in older fish. However, most Diplostomum species are known to have long life spans in their fish hosts, hence their accumulation with host size (Kennedy \& Burrough 1977, Chappell 1995, Nkwengulila 1995). No dead or moribund metacercariae were recorded during the present study, neither did Nkwengulila (1995), Mwita (2002) and Musiba (2004). Many workers have reported similar results (Chappell 1969, Kennedy and Burrough 1977); some however, have reported dead metacercariae in fish in the Palaearctic Region (Pennycuick 1971, Sweeting 1974, Nkwengulila 1995).

Frequency distribution of metacercariae Over dispersion of the metacercariae of Diplostomum species in their fish hosts is a common phenomenon (Sweeting 1974,
Kennedy 1984, Nkwengulila 1995, Mwita 2002, Musiba 2004). The decline in the degree of over dispersion coupled by a decline in mean intensity may be indicative of loss of the parasite population within the hosts, to which the explanation given above for the decline in mean intensity with age should apply.

The over dispersion of metacercariae in fish host populations has been attributed to heterogeneity in susceptibility, and uneven exposure of fish to infection that is a result of non-random distribution of cercariae in the habitat caused by aggregated distribution of snail intermediate hosts and chance effects (Anderson and Gordon 1982, Esch and Fernandez 1993, Bush et al. 2001).

\section{CONCLUSION}

The metacercariae of $D$. mashonense and Tylodelphys species were specific to $C$. gariepinus. Physiological differences may account for the absence of infection in the other clariids. Mutual exclusion or some factors governing the infection processes prevented the acquisition of large numbers of Tylodelphys 1 concurrent with large numbers of $D$. mashonense and / or Tylodelphys 2. Development of immunity, a short life span of the parasites and changes in recruitment rates with age of fish may explain the observed infection patterns. The over dispersed distribution of metacercariae in $C$. gariepinus is a result of inherent differences in susceptibility and uneven exposure of fish to infection.

\section{ACKNOWLEDGEMENTS}

The authors wish to thank the Lake Victoria Environment Management Project through the Fisheries Research Component coordinated by TAFIRI for financing this study.

\section{REFERENCES}

Anderson RM. and Gordon D.M 1982 Processes influencing the distribution of parasite numbers within host populations with special emphasis on parasite- 
induced host mortalities. Parasitology, 85: 373-398.

Betterton C 1974 Studies on the host specificity of eye fluke, Diplostomum spathaceum, in brown and rainbow trout. Parasitology, 69: 11-29.

Beverley-Burton M 1963 A new strigeid Diplostomum (T) mashonense n.sp. (Trematoda: Diplostomatidae) from the grey heron, Ardea cinerea L., in Southern Rhodesia with an experimental demonstration of part of the life cycle. Revue Zoologica Botanica Africa, 68: 291-306.

Brassard P, Rau ME and Curtis MA 1982 Parasite-induced susceptibility to predation in diplostomiasis. Parasitology 85: 495-501.

Burrough RJ 1978 The population biology of two species of eyefluke, Diplostomum spathaceum and Tylodelphys clavata, in roach and rudd. Journal of Fish Biology, 13: 19-32.

Bush AO, Fernandez JC, Esch GW and Seed JR 2001 Parasitism: The diversity and ecology of animal parasites. Cambridge University Press. 566 pp.

Chappell LH 1969 The parasites of the three-spined stickleback, Gasterosteus aculeatus, L., from a Yorkshire pond: Variation of the parasite fauna with sex and size of fish. Journal Fish of Biology, 1: 339-347.

Chappell LH 1995 The biology of diplostomatid eyeflukes of fishes. Journal of Helminthology, 69: 97-101.

Dubois G and Fain A 1956 Contribution a le'etude des Strigeida du Congo Belge. I. Bulletin de la Societé neuchâteloise des Sciences naturelles, 79: 12-38.

Esch GW and Fernandez JC 1993 A Functional Biology of Parasitism: ecology and evolutionary implications. Chapman and Hall. London. 337 pp.

Field JS and Irwin SWB 1994 The epidemiology, treatment and control of diplostomiasis on a fish farm in Northern Island. 87-100pp. In Pike AW. and Lewis JW (eds) Parasitic diseases of fish. Samara publishing (Ltd), Dyfed.
Kennedy CR and Burrough R 1977 The population biology of two species of eyefluke, Diplostomum gasterostei and Tylodelphys clavata, in perch. Journal of Fish Biology, 11: 619-633.

Kennedy CR 1984 The use of frequency distributions in an attempt to detect host mortality induced by infection of diplostomatid metacercariae. Parasitology, 89: 209-220.

Machado MH, Pavanelli GC and Takemoto RM 1996 Structure and diversity of endoparasitic infracommunities and the trophic level Pseudoplatystoma corruscans and Schizodon borelli (Osteichthyes) of the High Pavana River. Memorias do Instituto Oswaldo Cruz, Rio de Janeiro 91 (4): 441-448.

Mashego SN and Saayman JE 1989 Digenetic trematodes and cestodes of $C$. gariepinus in Lebowa South Africa. South African Journal of Wildlife Research, 11: 17-20.

Musiba M 2004 Some aspects of the life history of Diplostomum species (Digenea: Diplostomatidae) infecting Clarias species (Clariidae) of Lake Victoria. MSc Thesis, University of Dar es Salaam.

Mwita CJ 2002 Diversity and abundance of the parasite fauna of the catfish Clarias gariepinus (Burchell, 1822) (Clariidae) from the Mwanza Gulf, Lake Victoria. MSc. Thesis, University of Dar es Salaam. 157 pp.

Niewiadomska K 1996 The genus Diplostomum - taxonomy, morphology and biology. Acta Parasitologica, 41: 55-66.

Nkwengulila G 1995 Epidemiology and Taxonomy of Diplostomum species (Trematoda: Diplostomatidae) infecting fish of Llyn Tegid, North Wales and Ruvu basin-Tanzania. Ph.D. Thesis, University of Liverpool. 321pp.

Paperna I 1980 Parasites, infections and diseases of fish in Africa. CIFA. Tech. Pap. 7: 216pp.

Pennycuick L 1971 Quantitative effects of three species of parasites on a population 
of three-spined sticklebacks, Gasterosteus aculeatus. Journal of Zoological Society of London, 165: 143162.

Prudhoe S and Hussey CG 1977 Some parasitic worms in fresh-water fishes and fish predators from the Transvaal, South Africa. Zoologica Africana, 12: 113-148.

Shotter RA 1980 Aspects of the parasitology of the catfish (Clarias anguillaris, L.) from a river and a lake at Zaria, Kaduna state, Nigeria. Bulletin de $I F A N-T, \mathbf{4 2}$ : 837-859.
Stables JN and Chappell LH 1986 The epidemiology of diplostomiasis in farmed rainbow trout from northeast Scotland. Parasitology, 92: 699-710.

Sweeting RA 1974 Investigation into natural and experimental infections of freshwater fish by the common eyefluke, Diplostomum spathaceum Rud. Parasitology, 69: 291-300.

Zar JH 1996 Biostatistical analysis. Prentice-Hall, New Jersey. 718pp. 